\title{
Sheffield

\section{Analytical, experimental and numerical study of a graded honeycomb structure under in-plane impact load with low velocity}

GALEHDARI, S.A., KADKHODAYAN, M. and HADIDI-MOUD, Saeid

Available from Sheffield Hallam University Research Archive (SHURA) at:

http://shura.shu.ac.uk/10321/

This document is the author deposited version. You are advised to consult the publisher's version if you wish to cite from it.

\section{Published version}

GALEHDARI, S.A., KADKHODAYAN, M. and HADIDI-MOUD, Saeid (2015).

Analytical, experimental and numerical study of a graded honeycomb structure under in-plane impact load with low velocity. International Journal of Crashworthiness, 20

(4), 387-400.

\section{Copyright and re-use policy}

See http://shura.shu.ac.uk/information.html 


\title{
Analytical, experimental and numerical study of a graded honeycomb structure under in-plane impact load with low velocity
}

\author{
S.A.Galehdari ${ }^{1}$, M. Kadkhodayan, S. Hadidi-Moud
}

\begin{abstract}
Given the significance of energy absorption in various industries, light shock absorbers such as honeycomb structure under in-plane and out of plane loads have been in the core of attention. The purpose of this research is the analyses of graded honeycomb structure behavior (GHS) under in-plane impact loading and its optimization. Primarily, analytical equations for plateau stress and specific energy are represented, taking power hardening and elastic perfectly plastic models into consideration. For the validation and comparison of acquired analytical equations, the energy absorption of a graded honeycomb structure made of five different aluminum grades is simulated in ABAQUS/CAE. In order to validate the numerical simulation method in Abaqus, an experimental test has been conducted as the falling a weight with low velocity on a GHS. Numerical results retain an acceptable accordance with experimental ones with a 5.4\% occurred error of reaction force. For a structure with a specific kinetic energy, the stress-strain diagram is achieved and compared with the obtained analytical equations. The maximum difference between the numerical and analytical plateau stress for power hardening model (PHM) is $10.58 \%$. However, this value has been measured to be $38.78 \%$ for elastic-perfectly plastic model (EPPM). In addition, the numerical value of absorbed energy is compared to that of analytical method for two material models. The maximum difference between the numerical and analytical absorbed energy for PHM model is $6.4 \%$, while it retains the value of $48.08 \%$ for EPPM. Based on the conducted comparisons, the numerical and analytical results based on power hardening model are more congruent than elastic perfectly plastic model results. Applying Sequential Quadratic Programming method and genetic algorithm, the ratio of structure mass to the absorbed energy is minimized. According to the optimization results the structure capacity of absorbing energy increases by $18 \%$ compared to the primary model.
\end{abstract}

Keywords Graded honeycomb structure, in-plane impact load, power hardening, plateau stress, specific absorbed energy (SAE), optimization

\section{Nomenclature}

$\begin{array}{cl}G H S & \text { Graded Honeycomb Structure } \\ A & \text { Cross section area of GHS perpendicular to } \\ & \text { loading direction } \\ b & \text { Depth of GHS cell } \\ c & \text { Cell horizontal wall length } \\ d & \\ e & \text { Specific absorbed energy } \\ e_{f} & \text { Elongation } \\ K & \text { Coefficient of strain-hardening relation } \\ L & \text { Height of GHS } \\ l & \text { Cell inclined wall length } \\ l_{p} & \text { Plastic Hinge length } \\ l b & \text { Lower limits of the variables vector } \\ W & \text { Width of the GHS } \\ m & \text { GHS mass } \\ m_{c} & \text { Mass of each cell } \\ M_{p y} & \text { fully plastic moment based on EPPM } \\ M_{p u} & \text { fully plastic moment based on PHM } \\ M_{y} & \text { Initial yielding moment }\end{array}$

$\begin{array}{cl}n & \text { Strain-hardening index } \\ u & \text { Strain energy per unit mass } \\ U & \text { Strain energy } \\ \boldsymbol{u b} & \text { Upper limits of the variables vector } \\ \boldsymbol{X} & \text { Optimization design variables vector } \\ y & \text { Distance from neutral axis } \\ \sigma & \text { Stress tensor } \\ \sigma_{y} & \text { Yield stress } \\ \sigma_{u} & \text { Ultimate stress } \\ \sigma_{p} & \text { Plateau stress } \\ \varepsilon_{d} & \text { Locking strain } \\ \rho & \text { Density of honeycomb structure } \\ \rho_{s} & \text { Density of honeycomb structure material } \\ \rho^{*} & \text { Relative density of honeycomb structure } \\ \varepsilon_{c} & \text { Compressive strain } \\ \varphi & \text { Honeycomb cell's angle }\end{array}$

\footnotetext{
${ }^{1}$ Corresponding authour Email: ali.galehdari@gmail.com
} 


\section{Introduction}

With the noticeably rapid development in automotive, transportation and aeronautics engineering, analyzing the energy absorption capacity in structures have become an important field of research. In the last decade, various materials and structures with high specific absorbing energy such as graded honeycomb structure and thin vessel structures have been studied [1]. One of the most important characteristics of graded honeycomb structures is that by changing the geometrical parameters of the structure such as height, thickness, cell size and inner angles different mechanical characteristics could be obtained [2]. The most important purpose of this structure is reducing the effect of impact load by its distribution within a time period. The main characteristics of energy absorbing cellular structures are to absorb energy in an irreversible manner, reduce reactive load, undergo repeatable deformation mode, be compact, be light in weight and have higher specific energy absorption capacity, be inexpensive and easy to install. The common forms of cellular structures are (1) open cell structures in which cells are arranged in a two dimensional regular or irregular array and (2) closed cell structures in which plates are inter-connected and formed three dimensional, partially open or closed with regular or irregular shaped cells. Honeycomb structures are considered as one of the primary shock absorbers. These structures are widely used in automotive, aeronautics and packing industries. Scientifically speaking, banana peel which is a Functionally Graded Material (FGM) is a type of energy absorber [3]. Moreover, the human and bird bones are natural shock absorbers. The cancellous structure of bone leads to the absorption of applied shock as well as the reduction of bearing stress in joints. Aluminum honeycomb structures are well known energy absorbers and have wide applications in automobiles, aircrafts and packaging industries. They also provide a platform to expand knowledge of existing uniform wall open call structures to non-uniform cell wall graded structures [4]. Extensive research has been done in understanding the in-plane and out of plane behavior of honeycombs. Deqiang et al. [5] represented a FE model for analyzing the behavior of this type of structure under impact loads using LS-Dyna software. Song et al. [6] analyzed the dynamic pressure behavior of a 3D structure made of foam. An experimental test was also conducted through Voronoi arranging method. Another FE model was used by Song where the values of plateau stress and strain energy were obtained to investigate the influence of cells shape, impact load, relative density and strain hardening on the deformation mode and plateau stress. The results showed that the values of plateau stress and energy absorption increased with rising of cells irregularity. Asadi et al. [7] presented a simple FE model for the application of imploding in honeycomb structures. This model can be applied not only to the simple honeycomb, but also to the more complicated structures such as honeycomb structures with several layers or materials. The obtained numerical results showed an appropriate congruence with the experimental results. Liaghat et al. [8] optimized the honeycomb structure under compression loading and an optimization was conducted using both Matlab software and analytical methods. The optimization of structure cells with different geometries was also analyzed. Zou et al. [9] analyzed the in-plane dynamic destruction of regular honeycomb structures by FEM and compared the obtained plateau stresses by analytical and numerical methods to each other. They have also analyzed different mechanisms of structure cells deformation and have represented the stress-velocity diagrams. Ajdari et al. [10] analyzed the dynamic destruction behavior and the value of energy absorption in regular, irregular and FG honeycomb structures. They studied different modes of deformation and the value of energy absorption in these structures using FEM. Mohammad-Ali et al. [4, 11] simulated the behavior of graded honeycomb structure under impact load and presented an analytical equation for dynamic plateau stress corresponding to high velocities. The results of analytical equation were compared to those of numerical solution. In addition, to reduce the layer thickness in direction of panel sandwich thickness, the material hardness was also decreased. In another study, they studied the in-plane response of the graded structure under medium and high velocity impacts. Different critical energy absorbing characteristics, e.g. deformation modes, collapsing mechanism, crushing stress, locking strain and total energy absorbed have been discussed. In above studies the ideal elastic-perfectly plastic material model has been used to derive the plateau stress and specific energy of structure. However, comparison of numerical and analytical result shows high difference. In the current research, in order to reduce the difference, the plateau stress and specific energy of structure is driven based on power-hardening material model. To verify the driven equation, FE analysis is executed in ABAQUS. After that, according to driven equations, the specific energy of structure is optimized. Papka and Kyriakides [12-13] studied the load-displacement response of hexagonal-cell aluminum honeycombs as well as circular polycarbonate honeycombs under in-plane uniaxial loading. They observed various deformation patterns (modes), which were related to the particular ratio between the components of the applied displacements or forces. 


\section{Banana peel; a compact energy absorber}

The structures replicated from honeycombs are one of the prime candidates for reducing the impact in automobile, aerospace and packaging industries [14]. Figure 1 shows the cross sections of a banana peel. In technical terms, such kinds of materials are called Functionally Graded Materials (FGM). In FGM, the composition and structure gradually vary with depth, resulting in corresponding changes in the properties of the material. Observations show that such types of FGM structures are highly adaptive to all boundary and loading conditions defined by their environment. For example, the interior structure of a bone has an optimized shape with respect to the direction of principal stress and the magnitude of shear stress [15]. In a banana peel, one of the main objectives is to protect the internal soft core from external impacts.

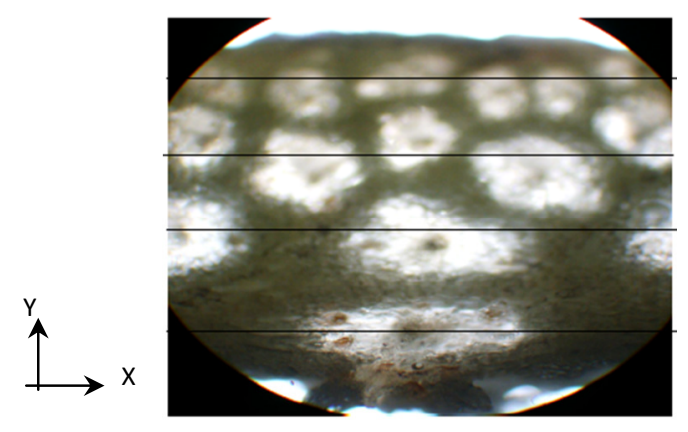

Figure 1. Cross section of a banana peel [3]

The arrangement of cells can be broken down into layers in the $X$ direction and the grading (variation in size) in the $Y$ direction (see Figure. 1). Four layers can be easily identified. These layers are indicated by horizontal lines. The 1st layer from top is composed of closely packed cells. The 2nd layer is composed of bigger cells with more spacing among them. This variation in the pattern continues until the last layer, where cells are widely dispersed. The graded structure shows that the stiffness changes with thickness. According to structural mechanics, two different sized cross sections with the same shape factor have different stiffness. The larger the cross section is, the lower the stiffness [16]. If a foreign object hits a banana peel from the top (or a banana falls on another object), the inner cells collapse first to protect the soft-core. This 'collapse mechanism' would flow up and layers would continue crushing until the whole structure is compromised. It is evident that such a 'collapse mechanism' allows structures to reduce the kinetic energy of the object over a finite period of time and the overall effect is a reduction in the impact load. The presence of fluid in the cells enhances the integrity and total energy absorption capability of the structure. The widely dispersed (biggest) cells in the bottom layer do not communicate structurally with one another. This informations lead to the hypothesis that the structure in a banana peel acts as a compact energy absorber. However, the banana peel structure is too complex to handle and therefore a few assumptions are made to redefine the structure without excessive deviation from the peel structure. It is assumed that the material is homogenous along the thickness, the cells have constant shape factor and are arranged in a uniform order. Figure 2 shows the modified peel structure and it may be called the graded honeycomb structure (GHS). The global response of the structure can be achieved by summing up the local response of individual rows. Due to the complexity of the peel structure and emphasis on extracting the cellular structural for investigating in-plane crushing behavior, the following assumptions are made to model the banana peel structure: (1) the cell wall material is homogenous, (2) the shape of the cells and their aspect ratios do not vary with thickness, and (3) the effect of fluid inside the cells is ignored. In addition, the cell arrangement depicted in Figure 1 more or less stays the same in the $\mathrm{Z}$ direction, which allows an inplane two dimensional analysis feasible. The quasi-static and low dynamic analysis for impact velocities up to $20 \mathrm{~m} / \mathrm{s}$ show that the peel graded structure has superior energy absorbing characteristics for a broader range of impact velocities in a restricted space as compared to regular honeycombs of constant wall thickness. A balanced response between the structure integrity and attenuation of reaction load is observed. At microscopic scale, the compacted cell shape keeps the impact effects low. A theoretical model was presented that correctly captured the crushing response of peel structure at static and low dynamic loadings [3]. According to the represented information, the semi honeycomb structure of the banana peel has the responsibility of protecting its core, in addition to several biological roles. Therefore, inspiring from a banana peel structure, a graded honeycomb structure is modeled. The integral characteristic of this type of absorber is its status of being graded. The variation of the stiffness of the structure from the hitting point to the protected body results in an increase of force transfer time. This duration, in case of being long, can be considered as a positive parameter for 
shock absorbers. Accordingly, a numerical, experimental and analytical study of graded honeycomb structure is conducted in this research.

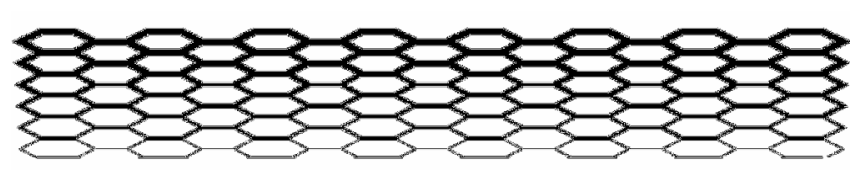

Figure 2. The modified peel structure [3]

\section{Mechanics of honeycomb structure}

A typical honeycomb cell with its parameters is shown in Figure 3. The behavior of honeycomb structure under compression loading can be determined using five modules including two Young modules $E_{1}$ and $E_{2}$, a shear module $G_{12}$, and two Poisson coefficients of $v_{12}$ and $v_{21}$.

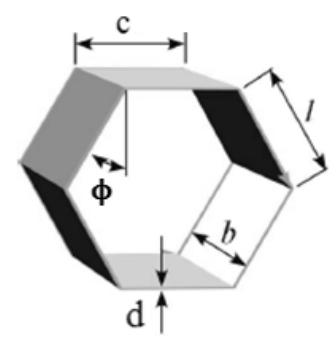

Figure 3. Honeycomb structure cell

Honeycomb structures transform in-plane kinetic energy into strain energy by crushing the rows. This strain energy is equal to plastic hinge energy and elastic one. It should be mentioned that the elastic strain energy is neglected due to its small amount compared to the plastic one. The most important parameters characterizing cellular material energy absorption properties are the plastic collapse stress generally known as the plateau stress and the relative density. The upper and lower bound theorems are used to determine the plateau stress. According to the upper bound theorem, an external load computed on the basis of an assumed mechanism, in which the forces are in equilibrium, is always greater than or equal to the true collapse load. On the other hand, the lower bound theorem says that an external load computed on the basis of an assumed distribution of internal forces, in which the forces are bounded by limit values and the forces are in equilibrium, is less than or equal to the true collapse load [17]. If a part of stress-strain diagram has a constant stress, it is called plateau stress. In fact, the value of plateau stress is not constant; however, its changes are negligible [18]. In deriving analytical equations, the value of $\sigma_{p}$ is considered as constant. So far the elastic-perfectly plastic model has been used to derive the plateau stress. In this study, due to the previously high difference between the numerical and analytical results, the power hardening model is used. The fully plastic moment of honeycomb wall is given by

$M_{p}=2 b \int_{0}^{\frac{d}{2}} y \sigma d y$

Based on the elastic-perfectly plastic model, the fully plastic moment can be obtained as

$M_{p y}=\frac{b \sigma_{y} d^{2}}{4}$

Considering the material model with the power hardening and by substituting $\sigma=K \varepsilon^{n}$ and $\varepsilon=\frac{2 y}{d} \varepsilon_{\max }$ [19] in Eq. (1) the corresponding fully plastic moment can be obtained 
$M_{p u}=\frac{b \sigma_{u} d^{2}}{2(n+2)}$

where $\sigma_{u}$ is the ultimate strength of the material of structure cell. Based on upper and lower theorem and using Eq. (2) the plastic-perfectly plastic plateau stress can be derived [17]

$\sigma_{p}=\frac{\sigma_{y} d^{2}}{2(c+l \sin \phi) l \sin \phi}$

The compressive load in the $Y$ direction is transferred to the inclined walls and they bend like a frame. The plastic analysis shows that six plastic hinges [20] are required to define the complete 'collapse mechanism' of a cell. Figure 4 shows the inclined wall undergoing angular rotation, $\psi$, with respect to its original position. An upper bound on the load acting on the wall is given by

$P=\sigma_{p}(c+l \sin \phi) b$

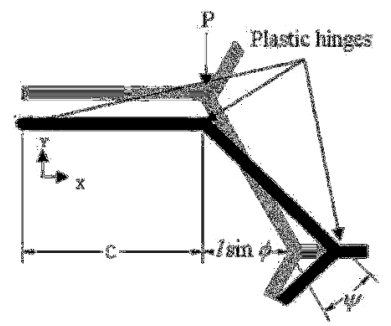

Figure 4. Plastic collapse of inclined walls in the $Y$ direction

For a cantilever beam subjected to load $\mathrm{P}$, the plastic hinge length $\mathrm{l}_{\mathrm{p}}$ is determined from the ultimate moment diagram at the point of collapse. The plastic hinge length at the end of the beam is given by

$\mathrm{l}_{\mathrm{p}}=\frac{\mathrm{l}}{4}\left(1-\frac{\mathrm{M}_{\mathrm{y}}}{\mathrm{M}_{\mathrm{p}}}\right)$

where $M y$ and $M_{p}$ are initial yielding and fully plastic moments, respectively. Initial yielding moment can be obtained as [21]

$M_{y}=\frac{b \sigma_{y} d^{2}}{6}$

Substituting Eqs (3) and (7) into Eq. (6), the plastic hinge length at the end of the beam for PHM is derived as

$l_{\mathrm{p}}=\frac{1}{4}\left(1-\frac{(\mathrm{n}+2) \sigma_{\mathrm{y}}}{3 \sigma_{\mathrm{u}}}\right)$

For $\frac{d}{l}<0.25$, the axial and shear deflections are small compared to bending deflections. Therefore, they do not significantly affect the plateau stress and bending moment considerably [17]. Plastic hinge length itself has little effect on the load but significantly changes the deformation geometry and hence the moment arm of the bending moment [22]. Therefore, only the plastic hinge length effect was taken into account to derive the plateau stress equation. The length of the plastic hinge was obtained by observing the values of bending moment, equivalent plastic strains and von Mises stress [23-24] at the integration points of the shell elements in the FE analysis. It was found to equal half the thickness of the cell wall. Hence, the moment arm $(l)$ is reduced to $l-d$. A lower bound on a collapse load is calculated by equating the internal negative moment on the cell wall to the external positive moment as shown in Figure 5 (Eq. 9). 


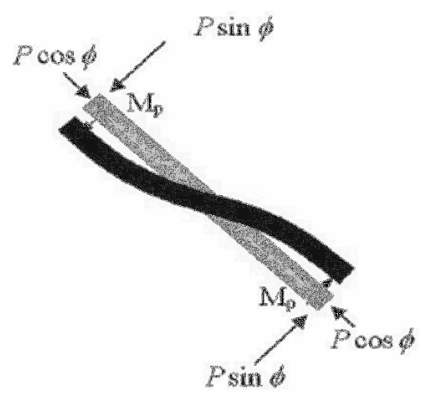

Figure 5. Internal and external bending moments on the inclined wall

$2 M_{p}=P(l-d) \sin \phi$

Substituting Eqs (3) and (5) into Eq. (9), the power hardening model plateau stress is derived as

$\sigma_{p}=\left(\frac{\sigma_{u}}{n+2}\right) \frac{d^{2}}{(c+l \sin \phi)(l-d) \sin \phi}$

The corresponding locking strain based on relative density can be calculated as [17]

$\rho=\frac{\left(\frac{d}{l}\right)\left(\frac{c}{l}+2\right)}{2\left(\sin (\phi)+\frac{c}{l}\right) \cos (\phi)} \rho_{s}$

It is noteworthy that $\rho^{*}$ is the ratio of structure cell density to the density of the material of the honeycomb structure $\left(\rho^{*}=\frac{\rho}{\rho_{s}}\right)$. In the above mentioned equation, $\rho_{s}$ is the density of the material of honeycomb structure. The porosity, which in fact is the pore volume, is $1-\frac{\rho}{\rho_{s}}$. This value is approximately equal to the locking strain $\varepsilon_{d}$ as [17]

$\varepsilon_{d}=1-\rho^{*}=1-\frac{\left(\frac{d}{l}\right)\left(\frac{c}{l}+2\right)}{2\left(\sin (\phi)+\frac{c}{l}\right) \cos (\phi)}$

It should be mentioned that by increasing the thickness of honeycomb cell wall, the locking strain becomes lower than that of the calculated value in the equation mentioned above; however, the exact value could be obtained through the experimental tests. Parameter $\varepsilon_{d}$ is the strain corresponding to the end of deformation in each row. In the following, it is assumed that the absorbed energy is equal to the strain energy of the entire structure. The equation of strain energy is

$U=\int\left(\int \sigma d \varepsilon\right) d V$

where $\sigma$ is the stress tensor applied to the structure, $\varepsilon$ is the strain tensor and $V$ is the volume of the structure. In order to calculate the strain energy per unit volume, the area below the surface of the stress-strain diagram is found, Figure. 5. Since the thickness of the cell walls is changing, the equations presented above can only capture the response of individual rows. Each row of the structure would confront deformation in plateau stress and locking strain. Therefore, considering $\sigma=\sigma_{p}$ and $\varepsilon=\varepsilon_{d}$ (the equal values of stress to plateau stress and strain to locking strain), strain energy per unit volume would be

$u=\sum_{i=1}^{6} \sigma_{p_{i}} \varepsilon_{d_{i}}$

In order to study the behavior of the entire structure, the analytical equations of all layers can be then summed up. According to the represented analytical equations, the plateau stress and locking strain diagram has been demonstrated in Figure. 6. 


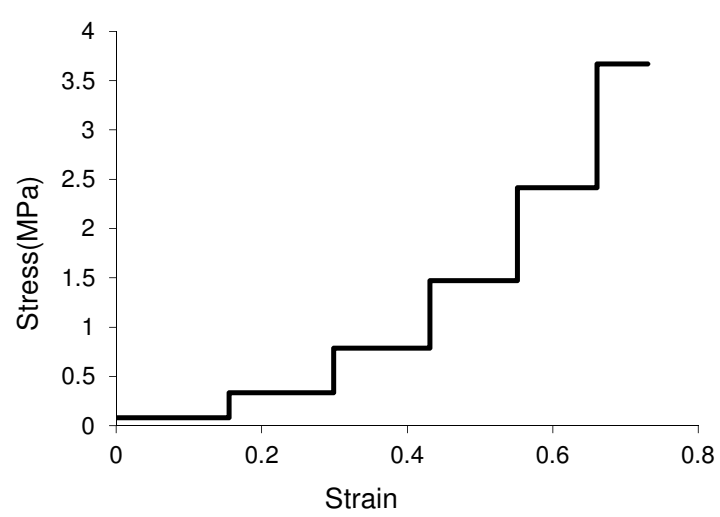

Figure 6. Analytical behavior of GHS in $Y$ direction, using Eqs (7) and (9)

Based on the obtained equations, the strain energy for the entire structure can be obtained as

$U=\int\left(\int \sigma_{p} d \varepsilon_{d}\right) d V=A L \sum_{i=1}^{6} \sigma_{p_{i}} \frac{\varepsilon_{d_{i}}}{6}$

where $\sigma_{p_{i}}$ is the plateau stress of each row for the material model with power hardening, $\varepsilon_{d_{i}}$ is the corresponding locking strain for each row, $A$ is the cross section of the structure perpendicular to the longitudinal direction and $L$ is the structure height in longitudinal direction (direction of collision). According to the dimensions of honeycomb, the energy equation of Eq. (15) can be rewritten as

$U=2 b l \cos (\phi)(15 c+16 l \sin (\phi)) \sum_{i=1}^{6} \sigma_{p_{i}} \varepsilon_{d_{i}}$

where $\sigma_{p_{i}}$ is calculated for power hardening model as

$\sigma_{p_{i}}=\left(\frac{\sigma_{u}}{n+2}\right) \frac{d_{i}{ }^{2}}{(c+l \sin (\phi))\left(l-d_{i}\right) \sin (\phi)}$

and for elastic perfectly plastic model as

$\sigma_{p_{i}}=\left(\frac{\sigma_{y}}{2}\right) \frac{d_{i}{ }^{2}}{(c+l \sin (\phi))\left(l-d_{i}\right) \sin (\phi)}$

The cell volume and mass are

$V_{c}=d b(4 l+2 c)$

$m_{c}=\rho_{s} d b(4 l+2 c)$

The structure has 6 rows and 15 cells in each row. Since the thickness of each row is different in this structure, the mass of entire structure is

$m=\rho_{s} b(32 l+23 c) \sum_{i=1}^{6} d_{i}$

An important parameter in design of energy absorbers is the specific energy of structure which is 
$e=\frac{U}{m}=\frac{2 b l \cos \phi(15 c+16 l \sin \phi) \sum_{i=1}^{6} \sigma_{p_{i}} \varepsilon_{d_{i}}}{\rho_{s} b(32 l+23 c) \sum_{i=1}^{6} d_{i}}$

A numerical analysis through FEM is conducted to validate the driven analytical results.

\section{Validation of Analytical Equations}

In order to validate the obtained analytical equations and comparing the result of equations based on two different material models, energy absorption of graded honeycomb structures made of five various grades of aluminum is simulated in ABAQUS/CAE. In each problem, the structure is applied to a specific kinetic energy. The FE model made of aluminum 1100-O of graded honeycomb structure is demonstrated in Figure. 7. Hourglass controlled, 4 nodes, reduced integration shell elements (S4R) are used to mesh the structure and rigid bilinear quadrilateral elements (R3D4) are used to mesh plate A and plate B. The GHS is a thin walled and the S4R element can be used. The boundary conditions are defined by constraining the discrete rigid plate, $\mathrm{A}$, to move only in the $\mathrm{Y}$ plane and by fixing all the rotational and translational degrees of freedom of the discrete rigid plate, B. Interaction properties are imposed using a general contact condition for contact of rows and surface to surface kinematic contact conditions between the top element based surface of the structure and the rigid plate, A. A penalty contact condition with friction tangential behavior is applied between the bottom element based surface of the structure and the rigid plate, B. In this module the coefficient of friction is equal to 0.2 . Individual rows are attached to each other by Tie module.

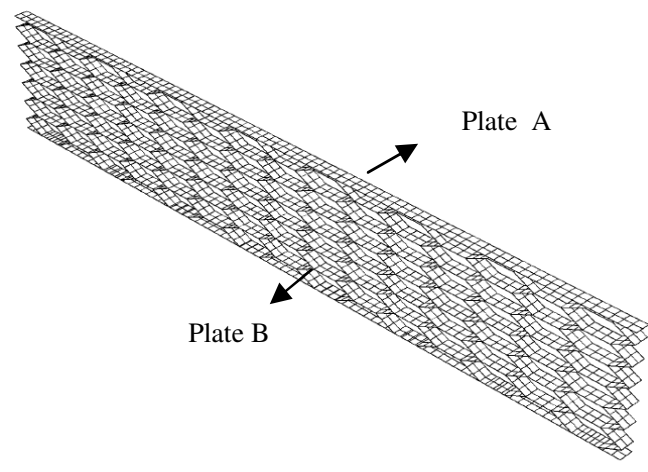

Figure 7. FE model of GHS

In this simulation, the plateau stress-locking strain diagram of the structure obtained from numerical solution is compared to analytical results for each material model. In addition, the numerical value of energy absorption is compared to that of analytical method for each material model. The geometrical characteristics of the structure are shown in Table 1. The material characteristics of utilized aluminums and the value of kinetic energy applied to the structure for power hardening model and elastic perfectly plastic model are represented in Tables 2 and 3, respectively. 
Table 1. Geometrical characteristics of the structure.

\begin{tabular}{ccc}
\hline $\begin{array}{c}\text { For AL-6061-O, } \\
\text { AL-7075,AL-5052, } \\
\text { AL 1100-O }\end{array}$ & For AL-2024 & $\begin{array}{c}\text { Geometric } \\
\text { parameter }\end{array}$ \\
\hline $2.7 \mathrm{~mm}$ & $2.7 \mathrm{~mm}$ & $c$ \\
$2.5 \mathrm{~mm}$ & $2.5 \mathrm{~mm}$ & $l$ \\
$0.35 \mathrm{~mm}$ & $0.6 \mathrm{~mm}$ & $d_{l}$ \\
$0.3 \mathrm{~mm}$ & $0.499 \mathrm{~mm}$ & $d_{2}$ \\
$0.25 \mathrm{~mm}$ & $0.399 \mathrm{~mm}$ & $d_{3}$ \\
$0.2 \mathrm{~mm}$ & $0.299 \mathrm{~mm}$ & $d_{4}$ \\
$0.15 \mathrm{~mm}$ & $0.2 \mathrm{~mm}$ & $d_{5}$ \\
$0.1 \mathrm{~mm}$ & $0.1 \mathrm{~mm}$ & $d_{6}$ \\
$63^{\circ}$ & $63^{\circ}$ & $\phi$ \\
\hline
\end{tabular}

Table 2. Material characteristics of different aluminums and kinetic energy applied to the structure for power hardening model

\begin{tabular}{ccccc}
\hline $\begin{array}{c}\text { Aluminum } \\
\text { grade }\end{array}$ & $\mathrm{K}$ (MPa) [25] & $\mathrm{n}[25]$ & $\begin{array}{c}\mathrm{S}_{\mathrm{ut}}(\mathrm{MPa}) \\
{[26]}\end{array}$ & $\begin{array}{c}\text { Applied } \\
\text { kinetic } \\
\text { energy }(\mathrm{J})\end{array}$ \\
\hline $1100-\mathrm{O}$ & 180 & 0.2 & 137 & 1.384 \\
$6061-\mathrm{O}$ & 205 & 0.2 & 150 & 0.555 \\
$5052-\mathrm{O}$ & 210 & 0.13 & 172 & 0.705 \\
$7075-\mathrm{O}$ & 400 & 0.17 & 290 & 1.185 \\
$2024-\mathrm{T} 4$ & 690 & 0.16 & 437 & 2.04 \\
\hline
\end{tabular}

Table 3. Material characteristics of different aluminums and kinetic energy applied to the structure for elastic-perfectly plastic model

\begin{tabular}{ccc}
\hline Aluminum grade & $\mathrm{S}_{\mathrm{y}}(\mathrm{MPa})[26]$ & Applied kinetic energy (J) \\
\hline $1100-\mathrm{O}$ & 34.47 & 0.784 \\
$6061-\mathrm{O}$ & 55.15 & 0.38 \\
$5052-\mathrm{O}$ & 89.63 & 0.6 \\
$7075-\mathrm{O}$ & 103.42 & 0.71 \\
$2024-\mathrm{T} 4$ & 324.05 & 2 \\
\hline
\end{tabular}

Due to the above mentioned material properties, the plastic behavior based on two mentioned material model is defined for each row individually. Velocity of the plate A is assigned to its reference point using predefined field. The finite element problem is solved by dynamic/explicit solver. 


\section{Experimental Test}

In order to validate the numerical simulation method in Abaqus software, an experimental test has been conducted as the falling a weight with low velocity on a graded honeycomb structure. Our experimental model is a 6061-O aluminum GHS. This structure has 6 rows with different thicknesses. The rows are formed by ramrod and matrix then glued to each other by Adhesive-Film. The thickness of $1^{\text {st }}$ to sixth rows is 1.6, 1.27, 1.016, 0.8125, 0.635 and $0.508 \mathrm{~mm}$, respectively. For this model $\mathrm{c}=15,1=12, \mathrm{~b}=28.5 \mathrm{~mm}$ and the height and width are 130 and $130 \mathrm{~mm}$, respectively. This structure is designed and produced according to the production facilities, as demonstrated in Figure 8.

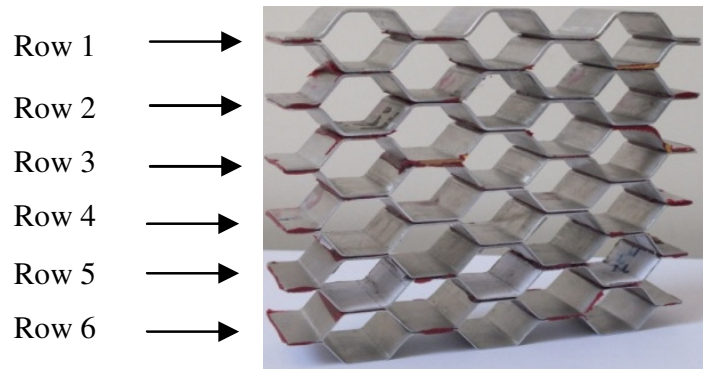

Figure 8. Testing sample of 6-row graded honeycomb structure

The rows of this structure have been formed by using punch and die and have been attached to each other by means of an adhesive film. To determinate the characteristics of this alloy for a more precise analysis, the materials of all of six thicknesses have been analyzed under a tension test using Santam machine. The stress-strain diagram of the uniaxial tensile tests on the standard specimens of AL-6061-O plate has been sketched to determine the each row material properties with the loading rate of $5 \mathrm{~mm} \cdot \mathrm{min}^{-1}$. The mechanical characteristics of each row have been listed in Table 4. It is noteworthy that the density and Poisson ratio of this aluminum are $2700 \mathrm{~kg} \cdot \mathrm{m}^{-3}$ and 0.33 , respectively. The obtained mechanical properties are used to define the material properties in finite element simulation. Low velocity impact test has been performed by drop hammer test device, Figure. 9. In this test, 99 Joule kinetic energy was applied to the GHS. A system of $9776.6 \mathrm{gr}$ has dropped from a 1.2 meter height. Due to the existence of several sources of loss such as friction, the velocity of the mass has been determined as $4.5 \mathrm{~m} / \mathrm{s}$ by a speedometer while striking the sample. Moreover, the acceleration of the block has been recorded during the impact to the structure and energy absorption by an accelerometer. This accelerometer records the mass acceleration in each 13 micro second and can measure maximum 100g. By multiplying the value of acceleration by the mass of the system, the reaction force of the structure can be computed, while the deformation of the structure is calculated by two numerical integrals from the acceleration value. Based on these results, force-displacement diagram was sketched. The fixture of test specimen is shown in Figure 10.

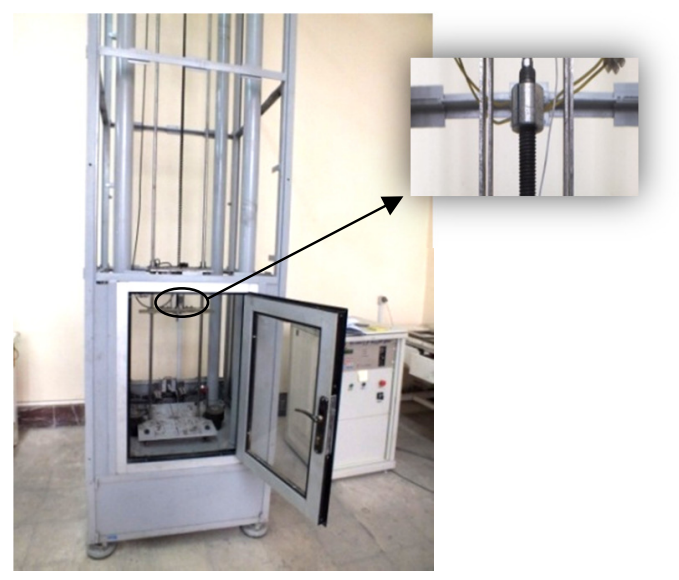

Figure 9. Drop-weight test device and the accelerometer 


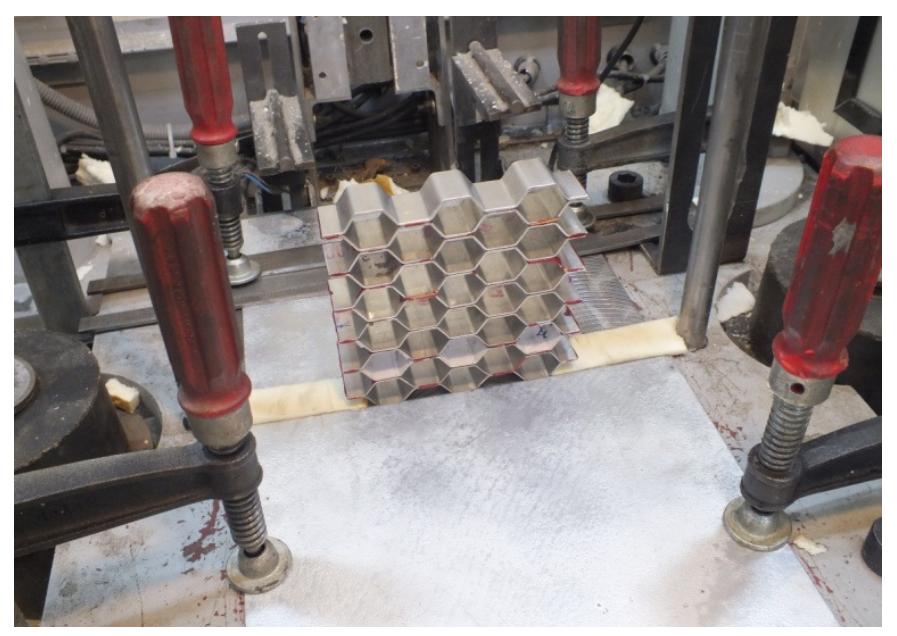

Figure 10. Test specimen fixture.

Table 4. Mechanical properties of different thickness of AL6061-O plate

\begin{tabular}{lllllll}
\hline Thickness $(\mathrm{mm})$ & $\mathrm{E}(\mathrm{GPa})$ & $\mathrm{n}$ & $\mathrm{K}(\mathrm{MPa})$ & $\mathrm{e}_{\mathrm{f}} \%$ & $\mathrm{~S}_{\mathrm{ut}}(\mathrm{MPa})$ & $\mathrm{S}_{\mathrm{y}}(\mathrm{MPa})$ \\
\hline 1.6 & 68.28 & 0.213 & 202.77 & 23.76 & 131.39 & 51.59 \\
1.27 & 66.98 & 0.245 & 242.66 & 25.142 & 141 & 51.92 \\
1.016 & 62.5 & 0.291 & 220.8 & 25.168 & 131 & 50.7 \\
0.8125 & 63.51 & 0.229 & 205.6 & 30.72 & 141 & 50 \\
0.635 & 64.3 & 0.247 & 228 & 27.06 & 134 & 48.15 \\
0.508 & 66.81 & 0.303 & 217.27 & 31.092 & 124 & 53 \\
\hline
\end{tabular}

\subsection{Numerical Simulation}

Regarding the material and geometrical characteristics of the structure, the mass and the velocity of the weight, loading and boundary conditions, the experimental test on the sample has been simulated in Abaqus Software. The FE model made of aluminum 6061-O of graded honeycomb structure is demonstrated in Figure. 11. The dropped mass and the structure base are modeled by plate A and B, respectively. Hourglass controlled, 8 nodes, reduced integration linear brick elements (C3D8R) are used to mesh the structure and rigid bilinear quadrilateral elements (R3D4) are used to mesh plate A and plate B, respectively. The structure wall is nearly thick here and the C3D8R element is used. The boundary conditions are defined by constraining the discrete rigid plate, A, to move only in the $\mathrm{Y}$ plane and by fixing all the rotational and translational degrees of freedom of the discrete rigid plate, B. Interaction properties are imposed using a general contact condition for contact of each rows and surface to surface kinematic contact conditions between the top element based surface of the structure and the rigid plate, A. A penalty contact condition with friction tangential behavior is applied between the bottom element based surface of the structure and the rigid plate, B. In this module based on test condition the coefficient of friction is considered equal to 0.6. The adhesive-film between the rows absorbs energy by converting the applied energy into strain energy. Hence, the glue is simulated by cohesive behavior using general contact interaction. Velocity of the plate A is assigned to its reference point using predefined field. Using the measured material properties the plastic behavior of AL- 60610 is defined using power hardening model for each row individually. The finite element problem is solved by dynamic/explicit solver. 


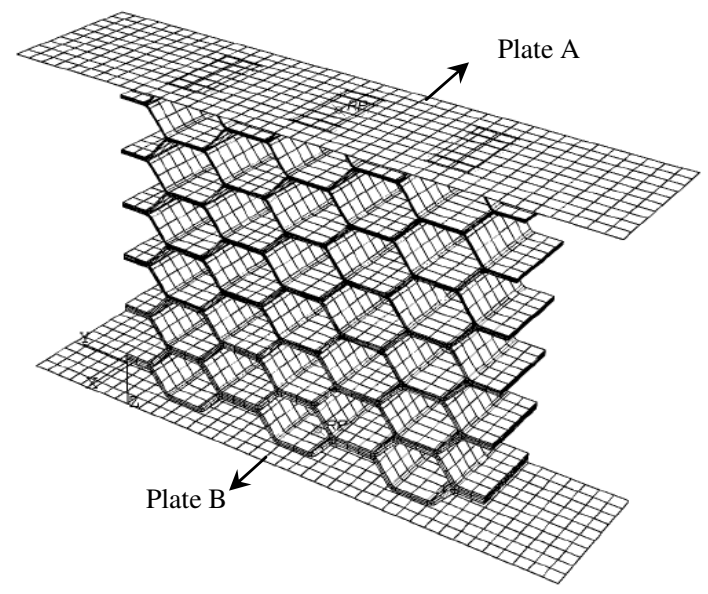

Figure 11. FE model of GHS

In this simulation, the reaction force-deformation diagram of the structure obtained from numerical solution is compared to the analytical and experimental results.

\section{Optimization}

The crashworthiness of structures under impact load can be improved by using optimization methods. Horstemeyer et al. [27] were used multi-objective optimization methods with finite element analysis in the lightweight design for sideimpact crashworthiness, considering two different criterions. Based on the results, the new injury-based design metric was much safer than the new energy absorption design metric. A genetic algorithm which is inspired from nature and is applied to study the optimization of complex systems is used here. In this approach, the search would continue by using genetic functions to the point of obtaining better or even the best samples. One of the most important characteristics of these algorithms is the possibility of parallel processing [28]. Another optimization algorithm is the $\mathrm{SQP}^{2}$ in which the objective function is minimized by non-linear constraints. This method is an iterative one for nonlinear optimization. SQP methods are used on problems for which the objective function and the constraints are twice continuously differentiable. SQP methods solve a sequence of optimization sub-problems, each of which optimizes a quadratic model of the objective subject to a linearization of the constraints. If the problem is unconstrained, then the method reduces to Newton's method for finding a point where the gradient of the objective vanishes. If the problem has only equality constraints, then the method is equivalent to applying Newton's method to the first-order optimality conditions, or Karush-Kuhn-Tucker conditions, of the problem [29]. In the current study, the initial structure consists of 6 cells in $Y$ direction and 15 cells in $X$ direction. The geometrical characteristics are mentioned in Table 1 . The structure is subjected by a rigid plane with a mass of $1 \mathrm{~kg}$ and $1.664 \mathrm{~ms}^{-1}$ initial velocity. The purpose of optimization here is the minimizing of the mass ratio to the absorbed energy of the structure. For this purpose, SQP and genetic algorithms are utilized in Matlab software with the objective function of the mass ratio to absorbed energy. According to Eq. (18), the objective function is the mass ratio to the absorbed energy of the structure and the design parameters are as given in Table 5. Moreover, the design variable vector $(\mathrm{X})$ with nine elements is defined in this table.

\footnotetext{
${ }^{2}$ Sequential Quadratic Programming
} 
Table 5. Design variables in optimization problem.

\begin{tabular}{cc}
\hline Design variable $(\boldsymbol{X})$ & Geometric parameter \\
\hline$x(1)$ & $d_{1}$ \\
$x(2)$ & $c$ \\
$x(3)$ & $l$ \\
$x(4)$ & $d_{2}$ \\
$x(5)$ & $d_{3}$ \\
$x(6)$ & $d_{4}$ \\
$x(7)$ & $d_{5}$ \\
$x(8)$ & $d_{6}$ \\
$x(9)$ & $\phi$ \\
\hline
\end{tabular}

For both algorithms the optimization problem is defined as:

$\min \left[\frac{m(\boldsymbol{X})}{U(\boldsymbol{X})}\right]$

$$
\text { s.t. } \quad m=0.0012 \mathrm{~kg} \text { and } \frac{x(i)}{x(3)}<0.25 \text {, for } i=1,3,4,5,6,7,8
$$

$\boldsymbol{l b} \leq \boldsymbol{X} \leq \boldsymbol{u b}$

The upper and lower limits of the variables are equal to the vectors $\boldsymbol{u b}$ and $\boldsymbol{l} \boldsymbol{b}$ as

$\boldsymbol{l b}=[0.0005 ; 0.0025 ; 0.002 ; 0.0004 ; 0.0003 ; 0.0002 ; 0.0001 ; 0.00005 ; 1.1](\mathrm{mm}, \mathrm{mm}, \mathrm{mm}, \mathrm{mm}, \mathrm{mm}, \mathrm{mm}, \mathrm{mm}, \mathrm{mm}, \mathrm{rad})$

$\boldsymbol{u} \boldsymbol{b}=[0.0006 ; 0.003 ; 0.0025 ; 0.0005 ; 0.0004 ; 0.0003 ; 0.0002 ; 0.0001 ; 1.3](\mathrm{mm}, \mathrm{mm}, \mathrm{mm}, \mathrm{mm}, \mathrm{mm}, \mathrm{mm}, \mathrm{mm}, \mathrm{mm}, \mathrm{rad})$

The optimization is conducted by two genetic and SQP algorithms and their results are compared to each other. In genetic algorithm, a generation of 30 and two stopping criteria are applied as

A) A maximum number of 100 generations.

B) The number of continuous generations without a change in optimum point reaches 20 .

A constraint and function tolerance of $10^{-9}$ is applied. In SQP method, the constraint and function tolerance equal to $10^{-6}$ and the start point equal to lower limit (lb) is considered.

\subsection{Numerical analysis}

To evaluate the optimization results, the obtained optimized structure is simulated by ABAQUS/CAE. The initial and optimized models are simulated and their energy absorption values are compared. The structure has a height of $17.16 \mathrm{~mm}$ and a width of $76.4 \mathrm{~mm}$. In both models, the material used in the structure is aluminum 1100-O with the density of 2700 $\mathrm{kg} / \mathrm{m}^{3}$. Geometrical parameters of this simulation are based on the genetic algorithm results. It is noteworthy that the power hardening material and elastic perfectly plastic model with aluminum is utilized and the rigid plane A collides to the structure with $1.791 \mathrm{~ms}^{-1}$ initial velocity. The rest of numerical simulation conditions are as mentioned in the section 4. 


\section{Results and Discussion}

In numerical simulation, the stress is calculated by dividing the reaction force of lower plate to its cross section and the strain is calculated by dividing the structure deformation to the initial length of the entire structure. According to the conducted numerical solution, the plateau stress-locking strain diagrams of two material models for aluminums with different grades are shown in Figure. 12. Moreover, the maximum difference between numerical and analytical results for power hardening material model and elastic-perfectly plastic model are represented in Tables 6 and 7, respectively. PHM denotes power hardening material model and EPPM denotes elastic-perfectly plastic material model in the following figures.

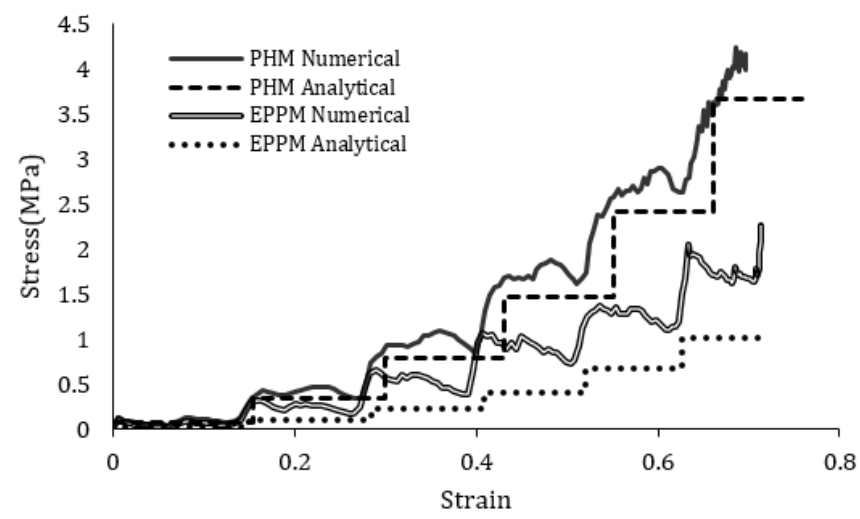

(a)

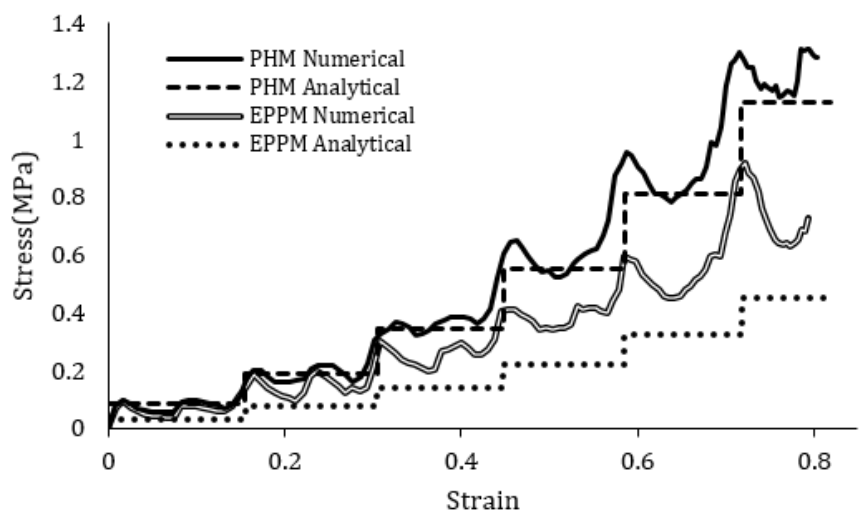

(b)

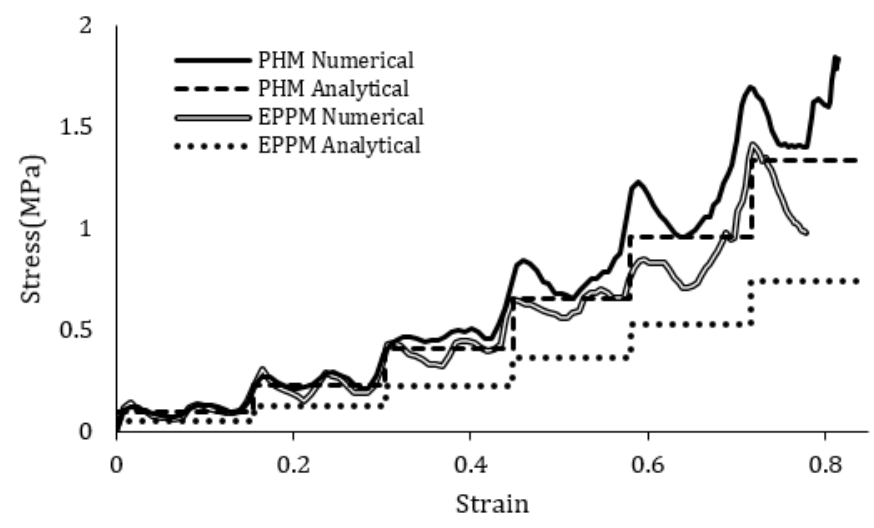

(c) 


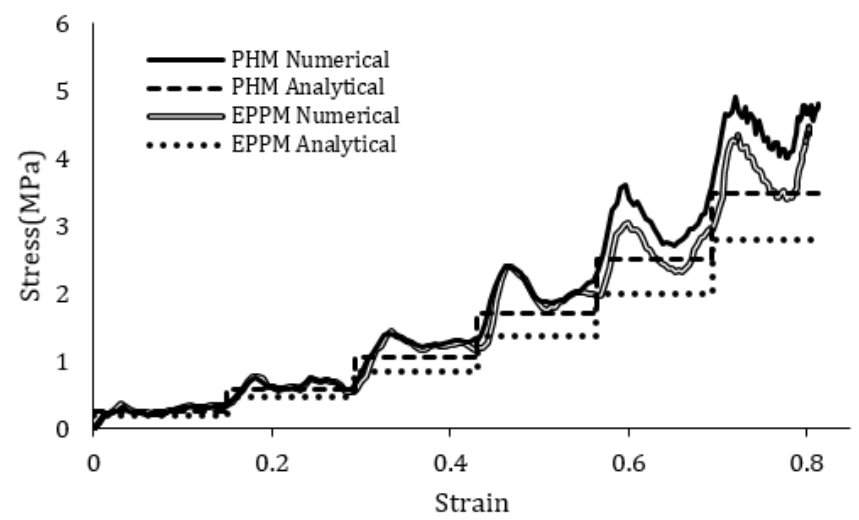

(d)

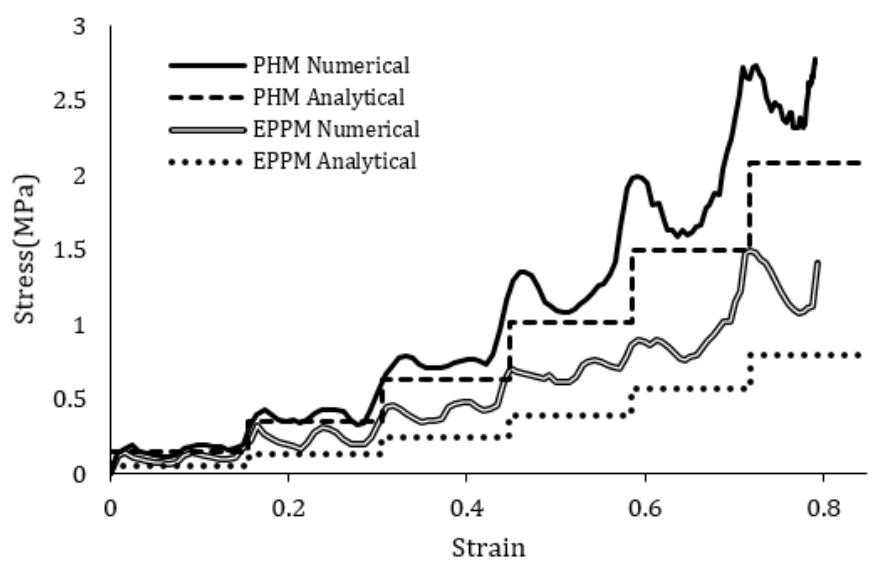

(e)

Figure 12. Stress-strain diagram for graded honeycomb structure made of different grades of aluminum, (a) 1100-O, (b) 6061-O, (c) 5052-O, (d) 7075-O and (e) 2024-T4

Table 6. Analytical and numerical plateau stress for different types of aluminum for PHM model

\begin{tabular}{cccc}
\hline $\begin{array}{c}\text { Aluminum } \\
\text { Grade }\end{array}$ & $\begin{array}{c}\text { Numerical } \\
\text { Stress (MPa) }\end{array}$ & $\begin{array}{c}\text { Analytical } \\
\text { Stress (Eq. 7) } \\
(\mathrm{MPa})\end{array}$ & $\begin{array}{c}\text { Maximum } \\
\text { Difference \% }\end{array}$ \\
\hline $1100-\mathrm{O}$ & 3.97 & 3.66 & 7.72 \\
$6061-\mathrm{O}$ & 1.14 & 1.13 & 0.8 \\
$5052-\mathrm{O}$ & 1.4 & 1.33 & 5 \\
$7075-\mathrm{O}$ & 2.31 & 2.08 & 9.9 \\
$2024-\mathrm{T} 4$ & 3.89 & 3.51 & 10.58 \\
\hline
\end{tabular}


Table 7. Analytical and numerical plateau stress for different types of aluminum for EPPM model

\begin{tabular}{cccc}
\hline $\begin{array}{c}\text { Aluminum } \\
\text { Grade }\end{array}$ & $\begin{array}{c}\text { Numerical } \\
\text { Stress } \\
(\mathrm{MPa})\end{array}$ & $\begin{array}{c}\text { Analytical Stress } \\
\text { (Eq. 4) }(\mathrm{MPa})\end{array}$ & $\begin{array}{c}\text { Maximum } \\
\text { Difference } \\
\%\end{array}$ \\
\hline $1100-\mathrm{O}$ & 1.65 & 1.01 & 38.78 \\
$6061-\mathrm{O}$ & 0.63 & 0.452 & 28.25 \\
$5052-\mathrm{O}$ & 0.987 & 0.735 & 25.53 \\
$7075-\mathrm{O}$ & 1.07 & 0.794 & 25.79 \\
$2024-\mathrm{T} 4$ & 3.42 & 2.79 & 18.42 \\
\hline
\end{tabular}

It is seen that the obtained values for plateau stress and locking strain from represented analytical equations for PHM model have more appropriate congruence rather than EPPM model with numerical results. The maximum difference between numerical and analytical of plateau stress for PHM model is $10.58 \%$; however for EPPM it is $38.78 \%$. Analytical equations based on power hardening decreases the difference between the numerical and analytical results compared with those based on elastic-perfectly plastic behavior. It, however, reasonably well captured the trend and pattern of the FE curve. With the conducted validation, the plateau stress equation based on PHM model can be utilized in deriving the equation of absorbed energy by the structure. Based on the kinetic energy applied to the structure, the analytical and numerical energy absorption values of structures with different aluminum grades for PHM model and EPPM model are compared in Figs. 13 and 14, respectively. In addition, the difference between numerical and analytical absorbed energy for two material models are represented in Tables 8 and 9.

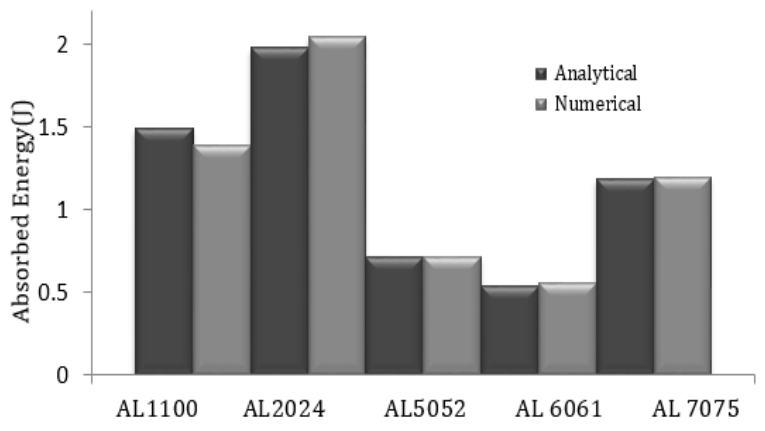

Figure 13. Comparing of analytical and numerical absorbed energy of GHS with different types of aluminum for PHM model

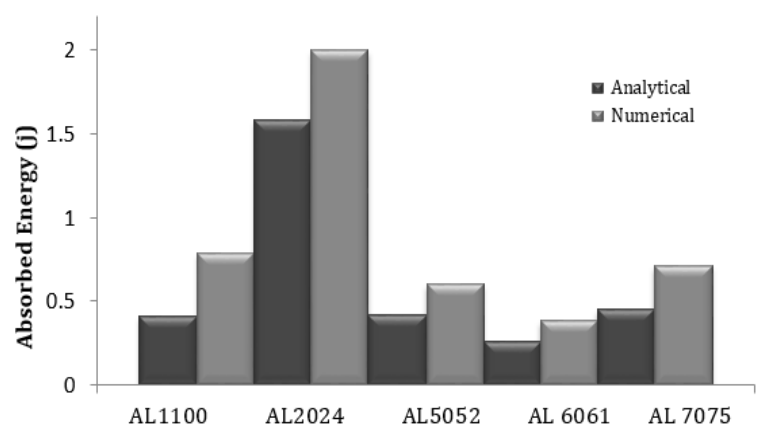

Figure 14. Comparing of analytical and numerical absorbed energy of GHS with different types of aluminum for EPPM model 
Table 8. Analytical and numerical absorbed energy for different types of aluminum for PHM model

\begin{tabular}{cccc}
\hline $\begin{array}{c}\text { Aluminum } \\
\text { Grade }\end{array}$ & $\begin{array}{c}\text { Numerical } \\
\mathrm{AE}(\mathrm{J})\end{array}$ & $\begin{array}{c}\text { Analytical AE } \\
(\text { Eq. 13) }(\mathrm{J})\end{array}$ & Difference \% \\
\hline $1100-\mathrm{O}$ & 1.385 & 1.48 & 6.4 \\
$6061-\mathrm{O}$ & 0.555 & 0.537 & 3.2 \\
$5052-\mathrm{O}$ & 0.705 & 0.704 & 3.2 \\
$7075-\mathrm{O}$ & 1.185 & 1.18 & 0.1 \\
$2024-\mathrm{T} 4$ & 2.04 & 1.97 & 0.4 \\
\hline
\end{tabular}

Table 9. Analytical and numerical absorbed energy for different types of aluminum for EPPM model

\begin{tabular}{cccc}
\hline $\begin{array}{c}\text { Aluminum } \\
\text { Grade }\end{array}$ & $\begin{array}{c}\text { Numerical } \\
\mathrm{AE}(\mathrm{J})\end{array}$ & $\begin{array}{c}\text { Analytical AE } \\
(\text { Eq.13) }(\mathrm{J})\end{array}$ & Difference \% \\
\hline $1100-\mathrm{O}$ & 0.784 & 0.407 & 48.08 \\
$6061-\mathrm{O}$ & 0.38 & 0.255 & 32.89 \\
$5052-\mathrm{O}$ & 0.6 & 0.415 & 30.8 \\
$7075-\mathrm{O}$ & 0.71 & 0.448 & 36.9 \\
$2024-\mathrm{T} 4$ & 2 & 1.57 & 21.3 \\
\hline
\end{tabular}

It is observed that the numerical and analytical results for PHM model indicate more proper congruence rather than EPPM model. The maximum difference between numerical and analytical of absorbed energy for PHM model is $6.4 \%$; however for EPPM it is $48.08 \%$. Figure 12 shows that the obtained analytical absorbed energy is properly close to numerical results, which shows the PHM model analytical equations can be utilized in deriving stress-strain diagram and finding the absorbed energy by the structure. Based on above comparison, the derived analytical equations for PHM model (Eqs. 7 and 13) shows the real behavior of material and then they are more useful and applicable than EPPM equations. Therefore the PHM equations can be used for SAE optimization of the graded honeycomb structure. In this simulation the hourglass and friction loss of energy are $0.28 \%$ and $0.27 \%$ of total internal energy, respectively. These amounts are lower than $5 \%$, so these energies can be neglected.

In the experimental test, the force-displacement diagram of the structure under the impact load with low velocity has been demonstrated in Figure 15, according to the obtained information from experimental test and numerical simulation. Furthermore, the deformed model of the structure after the experimental test and numerical simulation are compared in Figure 16.

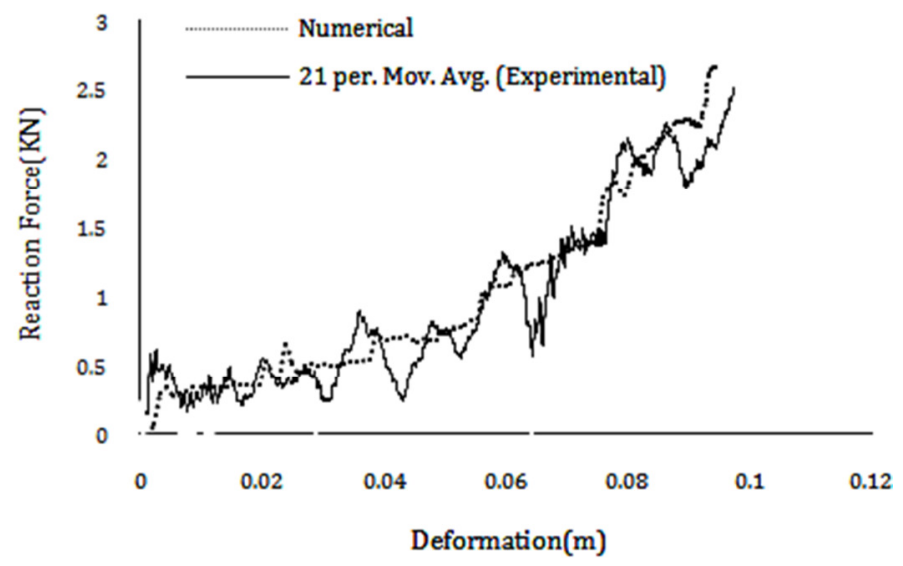

Figure 15. Force-displacement diagram of graded honeycomb structure for experimental test and numerical solution 


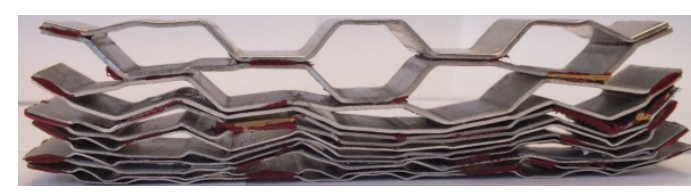

(a)

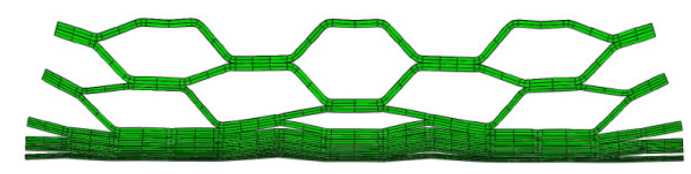

(b)

Figure 16. A deformed schema of the graded honeycomb structure obtained from (a) experimental test (b) numerical solution

Regarding Figures 15 and 16, numerical results retain an acceptable accordance with experimental results, in a way that the maximum error between the reaction force of experimental and numerical results is 5.4\%. Hence, the numerical simulation method and the applied parameters in this simulation are validated.

According to SAE optimization, the developed genetic algorithm stopped with the second stopping criteria after 31 iterations. The ratio of mass to absorbed energy is obtained as $7.475 \times 10^{-4} \mathrm{kgJ}^{-1}$. On the other hand, the SQP algorithm stopped after 20 iterations with the constraint and function tolerance criteria. The ratio of mass to absorbed energy is obtained as $8.088 \times 10^{-4} \mathrm{kgJ}^{-1}$. Optimization results based on two algorithms are represented in Table 10 .

Table 10. Obtained design parameters from genetic and SQP algorithms

\begin{tabular}{cccc}
\hline $\begin{array}{c}\text { Design } \\
\text { variable }\end{array}$ & $\begin{array}{c}\text { Initial } \\
\text { value }\end{array}$ & $\begin{array}{c}\text { Optimized } \\
\text { value by GA }\end{array}$ & $\begin{array}{c}\text { Optimized value } \\
\text { by SQP }\end{array}$ \\
\hline$l$ & $2.5 \mathrm{~mm}$ & $2.466 \mathrm{~mm}$ & $2.47 \mathrm{~mm}$ \\
$c$ & $2.7 \mathrm{~mm}$ & $2.96 \mathrm{~mm}$ & $2.94 \mathrm{~mm}$ \\
$t_{1}$ & $0.6 \mathrm{~mm}$ & $0.599 \mathrm{~mm}$ & $0.5826 \mathrm{~mm}$ \\
$t_{2}$ & $0.499 \mathrm{~mm}$ & $0.499 \mathrm{~mm}$ & $0.4868 \mathrm{~mm}$ \\
$t_{3}$ & $0.399 \mathrm{~mm}$ & $0.4 \mathrm{~mm}$ & $0.3868 \mathrm{~mm}$ \\
$t_{4}$ & $0.299 \mathrm{~mm}$ & $0.299 \mathrm{~mm}$ & $0.286 \mathrm{~mm}$ \\
$t_{5}$ & $0.2 \mathrm{~mm}$ & $0.1449 \mathrm{~mm}$ & $0.186 \mathrm{~mm}$ \\
$t_{6}$ & $0.1 \mathrm{~mm}$ & $0.0692 \mathrm{~mm}$ & $0.0899 \mathrm{~mm}$ \\
$\phi$ & $63^{\circ}$ & $54.43^{\circ}$ & $55.4^{\circ}$ \\
AE & $1.388 \mathrm{~J}$ & $1.6053 \mathrm{~J}$ & $1.4837 \mathrm{~J}$ \\
Iterations & - & 31 & 20 \\
\hline
\end{tabular}

By comparing the results obtained from genetic and SQP algorithms, it is seen that the ratio of mass to absorbed energy for the genetic algorithm is lower than that of the SQP, and then the former would be considered as the optimum choice. The absorbed energy by initial structure and optimum one are $1.388 \mathrm{~J}$ and $1.6053 \mathrm{~J}$, respectively. Consequently, the capacity of absorbing energy increases about $18 \%$. Based on the numerical simulation results, a history of the complete absorption of kinetic energy by GHS structure is demonstrated in Figure 17 for the initial and optimized models. 


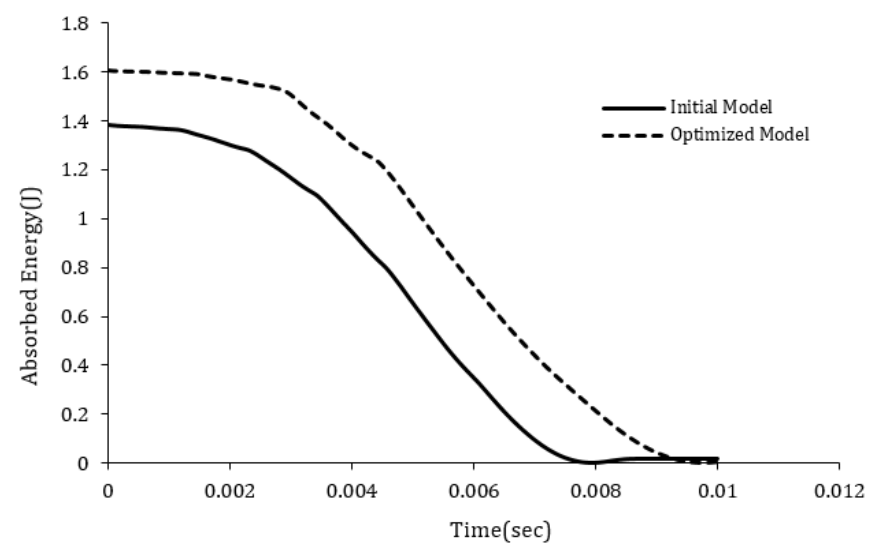

Figure 17. Diagram of kinetic energy changes for the initial and optimized model as a function of time

As it is observed, the initial structure absorbed the amount of $1.388 \mathrm{~J}$, while the optimized one completely absorbed $1.6053 \mathrm{~J}$ kinetic energy. The optimization results have appropriate congruence with simulation results. Increasing the duration of absorbing energy is an advantageous parameter in energy absorber design. In other words, the optimized structure could absorb more energy in a longer period of time. Hence, the optimization algorithm can increase both energy capacity and duration of energy absorbing.

The represented values of absorbed energy in sections 4 and 6 considering the dimensions of the modeled graded honeycomb structure are based on the banana peel structure. Applying optimization algorithms and the represented analytical equations with enlarging the dimensions of graded honeycomb structure and filling it with foam prove that the energy absorption can be increased. Using the introduced honeycomb structure, the analytical equations and optimization algorithm the energy absorption can be increased. This kind of energy absorber can be used for elevators, infant car seat and helicopter seat for improving the crashworthiness in emergency conditions.

\section{Conclusions}

Based on the obtained plateau stress equation and the locking strain equation, the specific absorbed energy equation for GHS was derived. To validate and compare the derived equations for two material models, a FE simulation on absorbing energy of GHS under in-plane impact load was performed. Comparison shows that the PHM model have more appropriate congruence rather than the EPPM one with numerical results. As previously mentioned, the maximum difference between the numerical and analytical plateau stress for PHM model is $10.58 \%$; however, for EPPM, this value is $38.78 \%$. Besides, the maximum difference between the numerical and analytical absorbed energy for PHM model is $6.4 \%$, while this value has been measured to be $48.08 \%$ for EPPM. Analytical equations based on power hardening reduce the difference between the numerical and analytical results compared with those based on EPPM. The obtained numerical results are of an acceptable accordance with experimental ones in such a way that the maximum measured error between the reaction force of experimental and numerical results is $5.4 \%$. According to the optimization results, the structure capacity of absorbing energy increases by $18 \%$ compared to that of the primary model. In fact, the graded status of the structure and the in-plane loading could reduce the applied impact load to the protected body and decrease the cost and physical damage compared to non-graded structure and out-of-plane loading. Generally and according to the increasing application of graded honeycomb structures, the represented analytical equations based on PHM model and optimization method can be utilized to reduce the ratio of structure mass to absorbed energy, in in-plane impact load conditions.

\section{References}

[1] Niknejad A and Liaghat G, Experimental study of Poly-orthan foam filler on hexagonal honeycomb structure behavior under axial load with constant rate (2011), $10^{\text {th }}$ Iran Aerospace conference (AERO2011), Tarbiat Modarres University. 
[2] Adibnazari S and Mehrabi H, Effect of cell size change on honeycomb structure equivalent mechanical property (2011), 10 ${ }^{\text {th }}$ Iran Aerospace conference (AERO2011), Tarbiat Modarres University.

[3] Muhammad A, Study of a compact energy absorber (2007), Ph.D. Thesis, Iowa State University.

[4] Muhammad A, Sun-il Kim, Tessa Matthews, Modeling of a compact functionally graded cellular structure: a finite element study for medium and high strain rates (2014), International Journal of Mechanic and Material Design, 10: 79-92.

[5] Deqiang S, Weihong Z and Yanbin W, Mean out-of-plane dynamic plateau stresses of hexagonal honeycomb cores under impact loadings (2010), Composite Structures, 92: 2609-2621.

[6] Song Y, Wang Z, Zhao L and Luo J, Dynamic crushing behavior of 3D closed-cell foams based on Voronoi random model (2010), Materials and Design, 31: 4281-4289.

[7] Asadi M, Shirvani H, Sanaei E and Ashmead M, A Simplified model to simulate crash behavior of honeycomb (2006), Proceedings of the International Conference on Advanced Design and Manufacture, China.

[8] Liaghat G and Sarailou H, Optimum design of honeycomb core under compression load (2010), Technical and Engineering Journal of Modarres, 37:73-81.

[9] Zou Z, Reid SR, Tan PJ, Li S, Harrigan JJ, Dynamic crushing of honeycombs and features of shock fronts (2009), International Journal of Impact Engineering, 36:165-167.

[10] Ajdari A, Nayeb-Hashemi H and Vaziri A, Dynamic crushing and energy absorption of regular, irregular and functionally graded cellular structures (2011), International Journal of Solids and Structures, 48:506-516.

[11] Muhammad A, Hoffman J, Clark J and Takak S, Modeling of impact response of composite graded structure (2011), IMECE2011, Denver, Colorado, U.S.A.

[12] Papka S D, Kyriakides S, In-plane compressive response and crushing of honeycomb (1994), Journal of Mechanics and Physics of Solids, 42:1499-1532.

[13] Papka S D, Kyriakides S, In-plane crushing of a polycarbonate honeycomb(1998), International Journal of Solids and Sructures, 35:239-267.

[14] Bitzer T, Honeycomb Technology: Materials, design, manufacturing, applications and testing (1997), Chapman and Hall, 1st edition.

[15] Przemieniecki KS, Theory of matrix structural analysis (1968), McGraw-Hill.

[16] Koch JC, The laws of bone architecture (1917), American Journal of Anatomy, 21:177-98.

[17] Gibson LJ and Ashby MF, Cellular Solids; Structures and Properties (1997), Cambridge University Press, 2nd edition.

[18] Lu G and Yu TX, Energy Absorption of structures and materials (2003), Wood head Publishing Limited \& CRC Press.

[19] Beer FP, Johnston ER, DeWolf JT, Mazurek DF ,Mechanics of materials (2012), McGraw-Hill.

[20] Yu TX and Zhang LC, Plastic bending: Theory and applications (1996), Series of Engineering Mechanics, World Scientific Pub Co Inc, Vol. 2.

[21] Rees DWA, Basic Engineering Plasticity (2006), Elsevier.

[22] Prager W and Hodge PG, Theory of perfectly plastic solids (1951), John Wiley.

[23] Kojic M and Bathe KJ, Inelastic analysis of solids and structures (2005), Springer.

[24] Khan AS and Huang S, Continuum theory of plasticity (1995), John Wiley.

[25] Kalpakjian S, Manufacturing Processes for engineering Materials (1991), 2nd edition, Adison-Wesley.

[26] Lyman T, Metals Handbook (1966), American Society of Metals, Ohio.

[27] Horstemeyer M, Ren XC, Fang H, Acar E and Wang PT , A comparative study of design optimization methodologies for side-impact crashworthiness using injury-based versus energy-based criterion (2009), International Journal of Crashworthiness, 14:125-138.

[28] Ggoldberg DE and Samadani MP, Engineering Optimization Via genetic algorithm (1986), 9th Conference of Electronic computation(ASCE), Alabama, University of Alabama, U.S.A.

[29] Bonnans, Frédéric J, Gilbert, Charles J, Lemaréchal, Claude, Sagastizábal and Claudia A, Numerical optimization: Theoretical and practical aspects (2006), Springer. 